\title{
Correlation between overweightness and the extent of coronary atherosclerosis among the South Caspian population
}

Maryam Nabati ${ }^{1}$, Mahmood Moosazadeh${ }^{2}$, Ehsan Soroosh ${ }^{3}$, Hanieh Shiraj ${ }^{1}$, Mahnaneh Gholami ${ }^{1}$ and Ali Ghaemian ${ }^{1,4^{*}}$ (D)

\begin{abstract}
Background: Reported effects of obesity on the extent of angiographic coronary artery disease(CAD) have been inconsistent. The present study aimed to investigate the relationships between the indices of obesity and other anthropometric markers with the extent of CAD.

Methods: This study was conducted on 1008 consecutive patients who underwent coronary angiography. Body mass index (BMI), waist circumference (WC), waist-to-hip ratio (WHR), and waist-to-height ratio (WHtR) were separately calculated for each patient. Extent, severity, and complexity of CAD were determined by the Gensini and SYNTAX scores.

Results: According to the results, there was a significant inverse correlation between the SYNTAX score with BMI $(r=-0.110 ; P<0.001)$, WC $(r=-0.074 ; P=0.018)$, and WHtR $(r=-0.089 ; P=0.005)$. Furthermore, a significant inverse correlation was observed between the Gensini score with BMI $(r=-0.090 ; P=0.004)$ and WHtR $(r=-0.065 ; P=$ 0.041). However, the results of multivariate linear regression analysis did not show any association between the SYNTAX and Gensini scores with the indices of obesity and overweight. On the other hand, the patients with an unhealthy WC had a higher prevalence of diabetes mellitus (DM) $(P=0.004)$ and hypertension (HTN) $(P<0.001)$, compared to the patients with healthy values. Coexistence of HTN and DM was more prevalent in subjects with an unhealthy WC and WHR, compared to that in those with healthy values ( $P=0.002$ and $P=0.032$, respectively).

Conclusion: It seems that the anthropometric indices of obesity are not the predictors of the angiographic severity of CAD. However, they are associated with an increased risk of cardiovascular risk factors and higher risk profile.
\end{abstract}

Keywords: Body mass index, BMI, coronary artery disease, Waist circumference

\section{Background}

Prevalence of obesity and overweight are increasing worldwide, and they are thought to be linked with the increased risk of several health problems, including type 2 diabetes, hypertension ( $\mathrm{HTN})$, coronary artery disease

\footnotetext{
* Correspondence: alighayemian@gmail.com

${ }^{1}$ Cardiovascular Research Center, Mazandaran University of Medical Sciences, Sari, Iran

${ }^{4}$ Mazandaran Heart Center, Artesh BLVD, Sari, Iran

Full list of author information is available at the end of the article
}

(CAD), and heart failure (HF) [1]. Recent studies have claimed that the measurements of abdominal obesity, including waist circumference (WC), waist-to-height ratio (WHtR), and waist-to-hip ratio (WHR), provide a superior tool for the discrimination between obesity-related cardiometabolic risk and BMI $[2,3]$.

Reported effects of obesity on the extent of angiographic CAD have been inconsistent [4]. In spite of the unfavorable effects of obesity on cardiovascular risk factors and increased prevalence of CAD among obese

(C) The Author(s). 2020 Open Access This article is licensed under a Creative Commons Attribution 4.0 International License, which permits use, sharing, adaptation, distribution and reproduction in any medium or format, as long as you give appropriate credit to the original author(s) and the source, provide a link to the Creative Commons licence, and indicate if changes were made. The images or other third party material in this article are included in the article's Creative Commons licence, unless indicated otherwise in a credit line to the material. If material is not included in the article's Creative Commons licence and your intended use is not permitted by statutory regulation or exceeds the permitted use, you will need to obtain permission directly from the copyright holder. To view a copy of this licence, visit http://creativecommons.org/licenses/by/4.0/ The Creative Commons Public Domain Dedication waiver (http://creativecommons.org/publicdomain/zero/1.0/) applies to the data made available in this article, unless otherwise stated in a credit line to the data. 
patients, some recent studies demonstrated an obesity paradox. It means that overweight and obese patients with known CAD may have a better prognosis than thinner subjects with CAD [5]. The present study aimed to investigate the relationships between BMI, WC, WHtR, WHR, and other anthropometric markers with the severity of CAD.

\section{Methods}

This historical cohort study was performed on 1008 consecutive patients who were admitted to our hospital and underwent coronary angiography due to acute coronary syndrome (ACS), suspected CAD due to chest pain, or the evidence of ischemia on non-invasive studies between September 2017 until October 2018. The study was conducted according to the guidelines of Helsinki Declaration and approved by the local Ethics Committee of our hospital. The patients were excluded from the study if referred for coronary angiography due to indications other than CAD such as valvular or congenital heart diseases, systemic infection, or any other comorbid conditions.

The demographics and medical history, including cardiovascular risk profiles, previous history of coronary artery bypass graft surgery, or percutaneous coronary intervention, were obtained from the subject medical record or face to face questionnaire. The HTN was defined as having a systolic blood pressure $\geq 140 \mathrm{mmHg}$, diastolic blood pressure $\geq 90 \mathrm{mmHg}$, or need for antihypertensive medications [6]. Diabetes mellitus (DM) was described according to the guidelines of the American Diabetes Association or need for taking oral hypoglycemic agents or insulin [7].

Family history (FH) of CAD was defined by the diagnosis of the disease in a male first-degree relative $<55$ years or female first-degree relative $<65$ years [8]. Hyperlipidemia (HLP) was characterized by a serum cholesterol level higher than $5.5 \mathrm{mmol} / \mathrm{L}$ and highdensity lipoprotein-cholesterol level lower than 1.0 $\mathrm{mmol} / \mathrm{L}$ in men or lower than $1.1 \mathrm{mmol} / \mathrm{L}$ in women [9]. History of smoking was determined by a face to face interview. Hormone replacement therapy was characterized by the combination treatment of estrogen plus progestogen, which was used to treat the symptoms of menopause. The individual's height (in meters) and weight (in kilograms) were measured, and BMI was calculated as body weight in kilograms divided by height in square metre.

According to the World Health Organization (WHO) classification, the patients in this study were categorized into three groups, namely normal weight (i.e., BMI lower than $25 \mathrm{Kg} / \mathrm{m}^{2}$ ), overweight (i.e., BMI of 25 to lower than $30 \mathrm{Kg} / \mathrm{m}^{2}$ ), and obese (i.e., BMI of $30 \mathrm{Kg} /$ $\mathrm{m}^{2}$ and above). The WC was determined as the smallest circumference between the umbilicus and xiphoid process. The patients were then categorized into two groups, including those with a healthy WC (i.e., $88 \mathrm{~cm}$ or lower in women and $102 \mathrm{~cm}$ or lower in men) and a high risk of WC (i.e., above $88 \mathrm{~cm}$ in women and 102 $\mathrm{cm}$ in men) [10].

Hip circumference was determined as the largest circumference around the buttocks posteriorly and the symphysis pubis anteriorly. The WHR was then calculated by dividing WC by hip circumference. According to the WHO classification, the subjects were categorized into two groups, including those with a healthy WHR (i.e., 0.9 or lower in men and 0.85 or lower in women) and a high risk of WHR (i.e., 0.91 or higher in men and 0.86 or higher in women). The WHtR was estimated by dividing WC by height. The patients were then categorized into two groups, namely nonobese cases with a WHtR lower than 0.5 and obese patients with a WHtR above 0.5 [11].

Diagnosis of ACS, including non-ST segment elevation myocardial infarction (NSTEMI), ST segment elevation myocardial infarction (STEMI), and unstable angina, was established according to the standards of the European Society of Cardiology (ESC) [12, 13]. The HF was also diagnosed according to the guidelines of the ESC [14]. All patients underwent transthoracic echocardiography within $24 \mathrm{~h}$ after hospitalization. Left ventricular ejection fraction (LVEF) was calculated by subtracting left ventricular end-systolic volume (LVESV) from left ventricular end-diastolic volume (LVEDV) divided by LVEDV using the biplane Simpson's method in apical four- and two-chamber views.

\section{Angiography}

All patients underwent coronary angiography by a Siemens AG (Medical Solutions; Erlangen, Germany). The severity of coronary atherosclerosis was calculated by quantitative coronary angiography method. The angiograms were analyzed by a group of cardiologists. At the end, an experienced interventional cardiologist blinded to the demographic and clinical data of the subjects reviewed the results and approved or corrected them. Significant CAD was defined as lesion $\geq 50 \%$ stenosis of the left main coronary artery and/or $\geq 70 \%$ stenosis of other major epicardial coronary arteries or branch vessels [15].

In this study the extent, severity, and complexity of CAD were assessed by the Gensini and SYNTAX scores. The Gensini score was calculated by giving a score to each coronary stenosis [16]. The patients were divided into two groups based on the Gensini score, including those with a low atherosclerotic burden (i.e., Gensini score $<7)$ and those with a high atherosclerotic burden (i.e., Gensini score $\geq 7$ ) [17]. 
Table 1 Anthropometric variables of the study population as divided by the tertiles of the SYNTAX score

\begin{tabular}{|c|c|c|c|c|c|c|c|c|}
\hline \multirow[t]{2}{*}{ Variables } & & \multirow[t]{2}{*}{ Total } & \multicolumn{4}{|c|}{ SYNTAX Score Group; n(\%) } & \multirow[t]{2}{*}{ Mean \pm SD } & \multirow[t]{2}{*}{$P$-Value } \\
\hline & & & $\overline{0-21}$ & $22-31$ & $>=32$ & P-value & & \\
\hline \multirow[t]{2}{*}{ Gender (frequency, percentile) } & Male & 606 & $513(84.7 \%)$ & 69(11.4\%) & $24(4.0 \%)$ & 0.250 & $9.29 \pm 10.34$ & $<0.001$ \\
\hline & Female & 402 & $354(88.1 \%)$ & $33(8.2 \%)$ & $15(3.7 \%)$ & & $6.98 \pm 9.97$ & \\
\hline \multirow[t]{5}{*}{ Age groups (years) } & $<40$ & 51 & $51(100.0 \%)$ & $0(.0 \%)$ & $0(.0 \%)$ & $<0.001$ & $2.09 \pm 3.82$ & $<0.001$ \\
\hline & $40-49$ & 175 & 164(93.7\%) & $9(5.1 \%)$ & $2(1.1 \%)$ & & $4.72 \pm 7.82$ & \\
\hline & $50-59$ & 385 & $328(85.2 \%)$ & $46(11.9 \%)$ & $11(2.9 \%)$ & & $8.51 \pm 9.70$ & \\
\hline & $60-69$ & 261 & $217(83.1 \%)$ & $31(11.9 \%)$ & $13(5.0 \%)$ & & $9.91 \pm 11.34$ & \\
\hline & $>=70$ & 136 & 107(78.7\%) & $16(11.8 \%)$ & $13(9.6 \%)$ & & $12.04 \pm 11.56$ & \\
\hline \multirow[t]{2}{*}{ HTN (frequency, percentile) } & Yes & 635 & $536(84.4 \%)$ & $69(10.9 \%)$ & $30(4.7 \%)$ & 0.083 & 9.2510 .59 & $<0.001$ \\
\hline & No & 372 & $331(89.0 \%)$ & $32(8.6 \%)$ & $9(2.4 \%)$ & & $6.81 \pm 9.43$ & \\
\hline \multirow[t]{3}{*}{ DM (frequency, percentile) } & Yes-Insulin & 108 & $86(79.6 \%)$ & $18(16.7 \%)$ & $4(3.7 \%)$ & 0.015 & $10.60 \pm 10.53$ & 0.001 \\
\hline & noninsulin & 283 & $234(82.7 \%)$ & $37(13.1 \%)$ & $12(4.2 \%)$ & & $9.48 \pm 10.63$ & \\
\hline & no & 617 & $547(88.7 \%)$ & $47(7.6 \%)$ & $23(3.7 \%)$ & & $7.47 \pm 9.92$ & \\
\hline \multirow[t]{2}{*}{ DM (frequency, percentile) } & Yes & 391 & $320(81.8 \%)$ & $55(14.1 \%)$ & $16(4.1 \%)$ & 0.004 & $9.79 \pm 10.60$ & $<0.001$ \\
\hline & No & 617 & $547(88.7 \%)$ & $47(7.6 \%)$ & $23(3.7 \%)$ & & $7.47 \pm 9.92$ & \\
\hline \multirow[t]{2}{*}{ CABG or PCI (frequency, percentile) } & Yes & 111 & 100(90.1\%) & $8(7.2 \%)$ & $3(2.7 \%)$ & 0.422 & $7.49 \pm 8.85$ & 0.344 \\
\hline & No & 833 & $755(85.5 \%)$ & $92(10.4 \%)$ & $36(4.1 \%)$ & & $8.47 \pm 10.42$ & \\
\hline \multirow[t]{2}{*}{ ACS or HF (frequency, percentile) } & Yes & 439 & $366(83.4 \%)$ & $53(12.1 \%)$ & $20(4.6 \%)$ & 0.105 & $9.58 \pm 10.62$ & 0.001 \\
\hline & No & 569 & $501(88.0 \%)$ & $49(8.6 \%)$ & $19(3.3 \%)$ & & $7.43 \pm 9.86$ & \\
\hline \multirow[t]{2}{*}{$\mathrm{FH}$ of premature CAD (frequency, percentile) } & Yes & 583 & $502(86.1 \%)$ & $61(10.5 \%)$ & $20(3.4 \%)$ & 0.654 & $7.94 \pm 10.009$ & 0.122 \\
\hline & No & 425 & $365(85.9 \%)$ & $41(9.6 \%)$ & $19(4.5 \%)$ & & $8.95 \pm 10.56$ & \\
\hline \multirow[t]{2}{*}{ Smoker (frequency, percentile) } & Yes & 157 & 138(87.9\%) & $16(10.2 \%)$ & $3(1.9 \%)$ & 0.381 & $8.60 \pm 9.16$ & 0.753 \\
\hline & No & 850 & $728(85.6 \%)$ & $86(10.1 \%)$ & $36(4.2 \%)$ & & $8.32 \pm 10.44$ & \\
\hline \multirow[t]{2}{*}{ Menopause (frequency, percentile) } & Yes & 289 & $247(85.5 \%)$ & $28(9.7 \%)$ & $14(4.8 \%)$ & 0.031 & $8.22 \pm 10.64$ & $<0.001$ \\
\hline & No & 113 & 107(94.7\%) & $5(4.4 \%)$ & $1(.9 \%)$ & & $3.82 \pm 7.10$ & \\
\hline \multirow[t]{2}{*}{ HRT (frequency, percentile) } & Yes & 78 & $71(91.0 \%)$ & $5(6.4 \%)$ & $2(2.6 \%)$ & 0.413 & $5.55 \pm 8.97$ & 0.012 \\
\hline & No & 930 & $796(85.6 \%)$ & $97(10.4 \%)$ & $37(4.0 \%)$ & & $8.60 \pm 10.32$ & \\
\hline \multirow[t]{2}{*}{ MI (frequency, percentile) } & Yes & 73 & $53(72.6 \%)$ & $13(17.8 \%)$ & $7(9.6 \%)$ & 0.002 & $13.06 \pm 11.58$ & $<0.001$ \\
\hline & No & 935 & $814(87.1 \%)$ & $89(9.5 \%)$ & $32(3.4 \%)$ & & $8.00 \pm 10.05$ & \\
\hline \multirow[t]{2}{*}{ ACS (frequency, percentile) } & Yes & 392 & $330(84.2 \%)$ & $44(11.2 \%)$ & $18(4.6 \%)$ & 0.389 & $9.45 \pm 10.48$ & 0.007 \\
\hline & No & 616 & $537(87.2 \%)$ & $58(9.4 \%)$ & $21(3.4 \%)$ & & $7.68 \pm 10.04$ & \\
\hline \multirow[t]{5}{*}{ Type of presentation (frequency, percentile) } & STEMI & 77 & $61(79.2 \%)$ & $11(14.3 \%)$ & $5(6.5 \%)$ & 0.001 & $11.79 \pm 10.07$ & $<0.001$ \\
\hline & Non STEMI & 51 & $36(70.6 \%)$ & 10(19.6\%) & $5(9.8 \%)$ & & $14.67 \pm 11.92$ & \\
\hline & Unstable Angina & 35 & $25(71.4 \%)$ & $8(22.9 \%)$ & $2(5.7 \%)$ & & $13.05 \pm 11.09$ & \\
\hline & Stable Angina & 707 & $622(88.0 \%)$ & $60(8.5 \%)$ & $25(3.5 \%)$ & & $7.71 \pm 9.99$ & \\
\hline & Others & 138 & 123(89.1\%) & $13(9.4 \%)$ & $2(1.4 \%)$ & & $6.31 \pm 9.26$ & \\
\hline \multirow[t]{2}{*}{ WC (cm) } & $<102$ or $<88$ & 367 & $311(84.7 \%)$ & $39(10.6 \%)$ & $17(4.6 \%)$ & 0.570 & $9.52 \pm 10.22$ & 0.007 \\
\hline & $>=102$ or $>=88$ & 641 & $556(86.7 \%)$ & $63(9.8 \%)$ & $22(3.4 \%)$ & & $7.71 \pm 10.21$ & \\
\hline \multirow[t]{2}{*}{ W/HR } & $<=0.9$ or $<=0.85$ & 28 & $26(92.9 \%)$ & $2(7.1 \%)$ & $0(.0 \%)$ & 0.467 & $6.14 \pm 7.81$ & 0.244 \\
\hline & $>0.9$ or $>0.85$ & 980 & $841(85.8 \%)$ & $100(10.2 \%)$ & $39(4.0 \%)$ & & $8.43 \pm 10.31$ & \\
\hline W/HtR & $<0.5$ & 60 & $51(85.0 \%)$ & $4(6.7 \%)$ & $5(8.3 \%)$ & 0.132 & $10.01 \pm 10.68$ & 0.201 \\
\hline & $>=0.5$ & 948 & $816(86.1 \%)$ & $98(10.3 \%)$ & $34(3.6 \%)$ & & $8.26 \pm 10.22$ & \\
\hline BMI $\left(\mathrm{kg} / \mathrm{m}^{2}\right)$ & $<25$ & 226 & $191(84.5 \%)$ & $23(10.2 \%)$ & $12(5.3 \%)$ & 0.111 & $9.64 \pm 10.41$ & 0.004 \\
\hline & $25-29$ & 465 & $392(84.3 \%)$ & $57(12.3 \%)$ & $16(3.4 \%)$ & & $8.77 \pm 10.17$ & \\
\hline & $>=30$ & 317 & $284(89.6 \%)$ & $22(6.9 \%)$ & $11(3.5 \%)$ & & $6.87 \pm 10.10$ & \\
\hline HLP (frequency, percentile) & Yes & 234 & 185(79.1\%) & $35(15.0 \%)$ & $14(6.0 \%)$ & 0.002 & $10.43 \pm 11.27$ & $<0.001$ \\
\hline & No & 774 & $682(88.1 \%)$ & $67(8.7 \%)$ & $25(3.2 \%)$ & & $7.74 \pm 9.84$ & \\
\hline
\end{tabular}

HTN Hypertension, DM Diabetes mellitus, CABG Coronary artery bypass graft, $P C I$ Percutaneous coronary intervention, $A C S$ Acute coronary syndrome, $H F$ Heart failure, $F H$ Family history, HRT Hormone replacement therapy, MI Myocardial infarction, STEMI ST-elevation myocardial infarction, Non STEMI Non ST-elevation myocardial infarction, WC Waist circumference, W/HR Waist to hip ratio, W/HtR Waist to height ratio, BMI Body mass index, HLP Hyperlipidemia, CAD Coronary artery disease 
Table 2 The distribution of anthropometric variables in patients with low SYNTAX score $(<21)$

\begin{tabular}{|c|c|c|c|c|c|c|}
\hline \multirow[t]{2}{*}{ Variables } & & \multirow[t]{2}{*}{ Total } & \multicolumn{4}{|c|}{ Syntax Score < 21; n(\%) } \\
\hline & & & 0 & $0.01-9$ & $9.1-21$ & $P$-value \\
\hline \multirow[t]{2}{*}{ Gender (frequency, percentile) } & Male & 513 & $200(39.0 \%)$ & 175(34.1\%) & $138(26.9 \%)$ & $<0.001$ \\
\hline & Female & 354 & $194(54.8 \%)$ & $102(28.8 \%)$ & $58(16.4 \%)$ & \\
\hline \multirow[t]{5}{*}{ Age groups (years) } & $<40$ & 51 & $35(68.6 \%)$ & $11(21.6 \%)$ & $5(9.8 \%)$ & $<0.001$ \\
\hline & 40-49 & 164 & $102(62.2 \%)$ & $39(23.8 \%)$ & $23(14.0 \%)$ & \\
\hline & $50-59$ & 328 & $139(42.4 \%)$ & $114(34.8 \%)$ & $75(22.9 \%)$ & \\
\hline & $60-69$ & 217 & $88(40.6 \%)$ & $70(32.3 \%)$ & $59(27.2 \%)$ & \\
\hline & $>=70$ & 107 & $30(28.0 \%)$ & $43(40.2 \%)$ & $34(31.8 \%)$ & \\
\hline \multirow[t]{2}{*}{ HTN frequency, percentile) } & Yes & 536 & $219(40.9 \%)$ & 183(34.1\%) & $134(25.0 \%)$ & 0.002 \\
\hline & No & 331 & $175(52.9 \%)$ & $94(28.4 \%)$ & $62(18.7 \%)$ & \\
\hline \multirow[t]{3}{*}{ DM (frequency, percentile) } & Yes-Insulin & 86 & $31(36.0 \%)$ & $27(31.4 \%)$ & $28(32.6 \%)$ & 0.031 \\
\hline & -noninsulin & 234 & $98(41.9 \%)$ & 75(32.1\%) & $61(26.1 \%)$ & \\
\hline & no & 547 & $265(48.4 \%)$ & $175(32.0 \%)$ & $107(19.6 \%)$ & \\
\hline \multirow[t]{2}{*}{ DM (frequency, percentile) } & Yes & 320 & $129(40.3 \%)$ & $102(31.9 \%)$ & $89(27.8 \%)$ & 0.011 \\
\hline & No & 547 & $265(48.4 \%)$ & $175(32.0 \%)$ & 107(19.6\%) & \\
\hline \multirow[t]{2}{*}{ CABG or PCI frequency, percentile) } & Yes & 100 & $36(36.0 \%)$ & $45(45.0 \%)$ & 19(19.0\%) & 0.011 \\
\hline & No & 755 & $353(46.8 \%)$ & $228(30.2 \%)$ & $174(23.0 \%)$ & \\
\hline \multirow[t]{2}{*}{ ACS or HF frequency, percentile) } & Yes & 366 & 134(36.6\%) & 143(39.1\%) & $89(24.3 \%)$ & $<0.001$ \\
\hline & No & 501 & $260(51.9 \%)$ & 134(26.7\%) & 107(21.4\%) & \\
\hline \multirow[t]{2}{*}{$\mathrm{FH}$ of premature CAD frequency, percentile) } & Yes & 502 & $240(47.8 \%)$ & 159(31.7\%) & $103(20.5 \%)$ & 0.150 \\
\hline & No & 365 & $154(42.2 \%)$ & $118(32.3 \%)$ & $93(25.5 \%)$ & \\
\hline \multirow[t]{2}{*}{ Smoker frequency, percentile) } & Yes & 138 & $49(35.5 \%)$ & $48(34.8 \%)$ & $41(29.7 \%)$ & 0.021 \\
\hline & No & 728 & $345(47.4 \%)$ & $229(31.5 \%)$ & $154(21.2 \%)$ & \\
\hline \multirow[t]{2}{*}{ Menopause frequency, percentile) } & Yes & 247 & $120(48.6 \%)$ & 79(32.0\%) & $48(19.4 \%)$ & 0.001 \\
\hline & No & 107 & $74(69.2 \%)$ & $23(21.5 \%)$ & $10(9.3 \%)$ & \\
\hline \multirow[t]{2}{*}{ HRT frequency, percentile) } & Yes & 71 & $44(62.0 \%)$ & $18(25.4 \%)$ & $9(12.7 \%)$ & 0.011 \\
\hline & No & 796 & $350(44.0 \%)$ & $259(32.5 \%)$ & $187(23.5 \%)$ & \\
\hline \multirow[t]{2}{*}{ MI (frequency, percentile) } & Yes & 53 & $9(17.0 \%)$ & $28(52.8 \%)$ & $16(30.2 \%)$ & $<0.001$ \\
\hline & No & 814 & $385(47.3 \%)$ & $249(30.6 \%)$ & $180(22.1 \%)$ & \\
\hline \multirow[t]{2}{*}{ ACS (frequency, percentile) } & Yes & 330 & $122(37.0 \%)$ & $126(38.2 \%)$ & $82(24.8 \%)$ & $<0.001$ \\
\hline & No & 537 & $272(50.7 \%)$ & $151(28.1 \%)$ & $114(21.2 \%)$ & \\
\hline \multirow[t]{5}{*}{ Type of presentation (frequency, percentile) } & STEMI & 61 & $9(14.8 \%)$ & $32(52.5 \%)$ & 20(32.8\%) & $<0.001$ \\
\hline & NSTEMI & 36 & $6(16.7 \%)$ & $16(44.4 \%)$ & $14(38.9 \%)$ & \\
\hline & Unstable Angina & 25 & $8(32.0 \%)$ & $8(32.0 \%)$ & 9(36.0\%) & \\
\hline & Stable Angina & 622 & $297(47.7 \%)$ & 192(30.9\%) & $133(21.4 \%)$ & \\
\hline & Others & 123 & $74(60.2 \%)$ & $29(23.6 \%)$ & $20(16.3 \%)$ & \\
\hline \multirow[t]{2}{*}{ WC (cm) } & $<102$ or $<88$ & 311 & $114(36.7 \%)$ & $113(36.3 \%)$ & $84(27.0 \%)$ & $<0.001$ \\
\hline & $>=102$ or $>=88$ & 556 & $280(50.4 \%)$ & $164(29.5 \%)$ & 112(20.1\%) & \\
\hline \multirow[t]{2}{*}{ W/HR } & $<=0.9$ or $<=0.85$ & 26 & $14(53.8 \%)$ & $5(19.2 \%)$ & $7(26.9 \%)$ & 0.369 \\
\hline & $>0.9$ or $>0.85$ & 841 & $380(45.2 \%)$ & $272(32.3 \%)$ & $189(22.5 \%)$ & \\
\hline \multirow[t]{2}{*}{ W/HtR } & $<0.5$ & 51 & $19(37.3 \%)$ & 17(33.3\%) & $15(29.4 \%)$ & 0.379 \\
\hline & $>=0.5$ & 816 & $375(46.0 \%)$ & $260(31.9 \%)$ & $181(22.2 \%)$ & \\
\hline \multirow[t]{3}{*}{ BMI $\left(\mathrm{kg} / \mathrm{m}^{2}\right)$} & $<25$ & 191 & $70(36.6 \%)$ & $67(35.1 \%)$ & $54(28.3 \%)$ & 0.004 \\
\hline & $25-29$ & 392 & $173(44.1 \%)$ & $125(31.9 \%)$ & $94(24.0 \%)$ & \\
\hline & $>=30$ & 284 & $151(53.2 \%)$ & $85(29.9 \%)$ & $48(16.9 \%)$ & \\
\hline \multirow[t]{2}{*}{ HLP (frequency, percentile) } & Yes & 185 & $80(43.2 \%)$ & $54(29.2 \%)$ & $51(27.6 \%)$ & 0.185 \\
\hline & No & 682 & $314(46.0 \%)$ & $223(32.7 \%)$ & $145(21.3 \%)$ & \\
\hline
\end{tabular}

HTN Hypertension, DM Diabetes mellitus, CABG Coronary artery bypass graft, $P C I$ Percutaneous coronary intervention, $A C S$ Acute coronary syndrome, $H F$ Heart failure, $F H$ Family history, HRT Hormone replacement therapy, MI Myocardial infarction, STEMI ST-elevation myocardial infarction, NSTEMI Non ST-elevation myocardial infarction, WC Waist circumference, W/HR Waist to hip ratio, W/HtR Waist to height ratio, BMI Body mass index, HLP Hyperlipidemia, CAD Coronary artery disease 
The total SYNTAX score was determined by summing up the individual scores for each lesion by an algorithm of the SYNTAX score accessible on the website of SYNTAX (http://www.syntaxscore.com) [16]. Again, the subjects were divided into three groups according to the SYNTAX score tertiles, including low: $\leq 21$, intermediate: $22-31$, and high $\geq 32$ [18]. Calculation of the Gensini and SYNTAX scores were evaluated by repeating the measurements in 20 randomly selected patients within $24 \mathrm{~h}$, and the intra-observer correlation coefficients were reported as 0.94 and 0.93 , respectively. An expert interventional cardiologist calculated the scores in all angiograms.

\section{Statistical analysis}

The continuous data were described as mean, standard deviation, as well as minimum and maximum values, and the categorical variables were explained by percentage and frequency. An independent t-test was used to compare the means between two groups in similar continuous. Moreover, dependent variable and analysis of variance were utilized to compare the means between more than two groups. The categorical variables were compared using the Chi-square and Fisher's exact tests.

Pearson's correlation coefficient was employed to assess the correlations between different anthropometric variables and extent of coronary atherosclerosis. Furthermore, multivariate linear regression analysis was used to determine the independent relationships between different anthropometric variables and the severity of coronary atherosclerosis. $P$-value $\leq 0.05$ was considered statistically significant. All statistical analyses were performed using SPSS software (version 16).

\section{Results}

A total of 1008 patients, including 606 males and 402 females, were investigated in the present study. The most common CAD risk factor was HTN (635 patients, 63\%), followed by having $\mathrm{FH}$ of premature CAD (583 patients, $57.8 \%$ ), DM (391 patients, 38.7\%), HLP (234 patients, 23.21\%), and cigarette smoking (157 patients, 15.57\%). Mean scores of age for male and female patients were $56.85 \pm 11.41$ and $57.21 \pm 10.77(26-90)$ years, respectively $(P=0.194)$. The mean BMI values were $27.49 \pm 4.07$ and $29.97 \pm 5.30 \mathrm{~kg} / \mathrm{m}^{2}$ for male and female patients, respectively $(P<0.001)$.

The mean SYNTAX score was higher in men, compared to that in women $(9.29 \pm 10.34$ and $6.98 \pm 9.97$, respectively; $\mathrm{P}<0.001)$. Furthermore, the elderly patients aged $\geq 70$ years had higher SYNTAX scores, compared to the subjects under 50 years of age $(12.04 \pm 11.56$ and $4.13 \pm 7.20$, respectively; $P<0.001)$. The SYNTAX score was significantly higher in diabetics in comparison to that in nondiabetics $(9.79 \pm 10.60$ and $7.47 \pm 9.92$, respectively; $\mathrm{P}<0.001)$. In addition, the SYNTAX score was significantly higher in hypertensives than that in normotensive patients $(9.25 \pm 10.59$ and $6.81 \pm 9.43$, respectively; $P<0.001)$. Table 1 tabulates the clinical and demographic variables of the study population based on the SYNTAX score tertiles.

Among the patients with the SYNTAX score $<21$, male subjects had a higher prevalence of the scores between 9.1 and 21, compared to female patients $(26.9 \%$ vs. 16.4\%; Table 2; $P<0.001)$. Regarding the Gensini score, it was significantly higher in men than that in women $(28.13 \pm 28.21$ vs. $21.32 \pm 27.86$, respectively; $\mathrm{P}<$ $0.001)$, higher in hypertensives than that in normotensive subjects $(27.64 \pm 28.91$ vs. $21.56 \pm 26.70$, respectively; $P=0.01$ ), and higher in hyperlipidemics than that in normolipidemics (31.11 \pm 31.65 vs. $23.71 \pm 26.93$; Table 3 ; $P<0.001)$. Severity of CAD expressed by the SYNTAX and Gensini scores in different age groups categorized by sex is represented in Figs. 1 and 2 .

Pearson's correlation coefficient was used to assess the association between the different anthropometric variables and Gensini and SYNTAX scores. According to the results, there was a significant direct association between the SYNTAX score and age $(r=0.250 ; P<0.001)$ and a significant inverse correlation between the SYNTAX score with BMI $(\mathrm{r}=-0.110 ; P<0.001)$, LVEF $(\mathrm{r}=-0.248 ; P<$ $0.001)$, WC $(\mathrm{r}=-0.074 ; P=0.018)$, WHR $(\mathrm{r}=-0.009 ; P=$ $0.780)$, and WHtR $(\mathrm{r}=-0.089 ; P=0.005)$. Furthermore, there was a significant direct association between the Gensini score and age $(r=0.240 ; P<0.001)$ and a significant inverse correlation between the Gensini score with BMI $(\mathrm{r}=-0.090 ; P=0.004), \operatorname{LVEF}(\mathrm{r}=-0.253 ; P<0.001)$, and WHtR $(\mathrm{r}=-0.065 ; P=0.041)$.

Then, multivariate linear regression analysis was conducted to modulate the confounding effects of other variables on the SYNTAX and Gensini scores (Tables 4 and 5). These analyses did not show any association between the SYNTAX and Gensini scores with the indices of obesity and overweightness.

In the present study, the prevalence rate of DM was reported as $38.8 \%$. The patients with an unhealthy WC had a higher prevalence of DM, compared to the subjects with a healthy WC $(42.1 \%$ vs. $33 \% ; P=0.004)$. In addition, the prevalence rate of HTN was reported to be $63 \%$. The patients with an unhealthy WC had a higher prevalence of $\mathrm{HTN}$, compared to the subjects with a healthy WC $(68.6 \%$ vs. $53.1 \%$; $P<0.001)$. Moreover, the obese and overweight patients had a higher prevalence of HTN, compared to the subjects with a normal weight (68.5\% vs. 58.4 and $61.5 \%$ vs. $58.4 \%$, respectively; $P=$ 0.038). Coexistent HTN and DM were observed in 290 cases $(28.8 \%)$, and this coexistence was more prevalent in patients with an unhealthy WC and WHR, compared to that in subjects with healthy values $(32.1 \%$ vs. $22.9 \%$, 
Table 3 Anthropometric variables of the study population as divided by Gensini score groups

\begin{tabular}{|c|c|c|c|c|c|c|c|}
\hline \multirow[t]{2}{*}{ Variables } & & \multirow[t]{2}{*}{ Total } & \multicolumn{3}{|c|}{ Gensini score; n(\%) } & \multirow[t]{2}{*}{ Mean \pm SD } & \multirow[t]{2}{*}{$\boldsymbol{P}$-Value } \\
\hline & & & $<7$ & $>=7$ & $\boldsymbol{P}$-value & & \\
\hline \multirow[t]{2}{*}{ Gender (frequency, percentile) } & Male & 606 & $155(25.6 \%)$ & $451(74.4 \%)$ & $<0.001$ & $28.13 \pm 28.21$ & $<0.001$ \\
\hline & Female & 402 & 159(39.6\%) & $243(60.4 \%)$ & & $21.35 \pm 27.86$ & \\
\hline \multirow[t]{5}{*}{ Age groups (years) } & $<40$ & 51 & $33(64.7 \%)$ & 18(35.3\%) & $<0.001$ & $6.91 \pm 10.48$ & $<0.001$ \\
\hline & $40-49$ & 175 & $83(47.4 \%)$ & $92(52.6 \%)$ & & $14.97 \pm 19.16$ & \\
\hline & $50-59$ & 385 & $109(28.3 \%)$ & $276(71.7 \%)$ & & $27.45 \pm 29.35$ & \\
\hline & $60-69$ & 261 & $66(25.3 \%)$ & 195(74.7\%) & & $27.68 \pm 29.61$ & \\
\hline & $>=70$ & 136 & 23(16.9\%) & 113(83.1\%) & & $35.80 \pm 30.03$ & \\
\hline \multirow[t]{2}{*}{ HTN (frequency, percentile) } & Yes & 635 & $169(26.6 \%)$ & $466(73.4 \%)$ & $<0.001$ & $27.64 \pm 28.91$ & 0.001 \\
\hline & No & 372 & $145(39.0 \%)$ & $227(61.0 \%)$ & & $21.56 \pm 26.70$ & \\
\hline \multirow[t]{3}{*}{ DM (frequency, percentile) } & Yes-Insulin & 108 & $26(24.1 \%)$ & $82(75.9 \%)$ & 0.009 & $31.85 \pm 31.30$ & $<0.001$ \\
\hline & noninsulin & 283 & $74(26.1 \%)$ & 209(73.9\%) & & $29.65 \pm 31.18$ & \\
\hline & no & 617 & $214(34.7 \%)$ & $403(65.3 \%)$ & & $22.37 \pm 25.79$ & \\
\hline \multirow[t]{2}{*}{ DM (frequency, percentile) } & Yes & 391 & $100(25.6 \%)$ & $291(74.4 \%)$ & 0.002 & $30.26 \pm 31.19$ & $<0.001$ \\
\hline & No & 617 & $214(34.7 \%)$ & $403(65.3 \%)$ & & $22.37 \pm 25.79$ & \\
\hline \multirow[t]{2}{*}{ CABG or $\mathrm{PCl}$} & Yes & 111 & $27(24.3 \%)$ & $84(75.7 \%)$ & 0.098 & $25.69 \pm 30.29$ & 0.881 \\
\hline & No & 883 & $283(32.0 \%)$ & $600(68.0 \%)$ & & $25.26 \pm 27.82$ & \\
\hline \multirow[t]{2}{*}{ ACS or HF } & Yes & 439 & $107(24.4 \%)$ & $332(75.6 \%)$ & $<0.001$ & $29.46 \pm 30.54$ & 0.000 \\
\hline & No & 569 & $207(36.4 \%)$ & $362(63.6 \%)$ & & $22.32 \pm 25.97$ & \\
\hline \multirow[t]{2}{*}{ FH of premature CAD (frequency, percentile) } & Yes & 583 & $188(32.2 \%)$ & $395(67.8 \%)$ & 0.379 & $24.86 \pm 28.71$ & 0.456 \\
\hline & No & 425 & $126(29.6 \%)$ & $299(70.4 \%)$ & & $26.20 \pm 27.64$ & \\
\hline \multirow[t]{2}{*}{ Smoker (frequency, percentile) } & Yes & 157 & $37(23.6 \%)$ & $120(76.4 \%)$ & 0.025 & $26.63 \pm 25.69$ & .554 \\
\hline & No & 850 & $277(32.6 \%)$ & $573(67.4 \%)$ & & $25.18 \pm 28.72$ & \\
\hline \multirow[t]{2}{*}{ Menopause (frequency, percentile) } & Yes & 289 & $96(33.2 \%)$ & 193(66.8\%) & $<0.001$ & $24.75 \pm 30.37$ & $<0.001$ \\
\hline & No & 113 & $63(55.8 \%)$ & $50(44.2 \%)$ & & $12.66 \pm 17.39$ & \\
\hline \multirow[t]{2}{*}{ HRT (frequency, percentile) } & Yes & 78 & $34(43.6 \%)$ & $44(56.4 \%)$ & 0.014 & $16.58 \pm 20.28$ & 0.004 \\
\hline & No & 930 & $280(30.1 \%)$ & $650(69.9 \%)$ & & $26.17 \pm 28.71$ & \\
\hline \multirow[t]{2}{*}{ MI (frequency, percentile) } & Yes & 73 & $8(11.0 \%)$ & $65(89.0 \%)$ & $<0.001$ & $40.80 \pm 32.14$ & $<0.001$ \\
\hline & No & 935 & $306(32.7 \%)$ & $629(67.3 \%)$ & & $24.23 \pm 27.59$ & \\
\hline \multirow[t]{2}{*}{ ACS (frequency, percentile) } & Yes & 392 & $95(24.2 \%)$ & $297(75.8 \%)$ & $<0.001$ & $29.00 \pm 29.88$ & 0.001 \\
\hline & No & 616 & 219(35.6\%) & $397(64.4 \%)$ & & $23.15 \pm 26.95$ & \\
\hline \multirow[t]{5}{*}{ Type of presentation (frequency, percentile) } & STEMI & 77 & $9(11.7 \%)$ & $68(88.3 \%)$ & $<0.001$ & $33.51 \pm 27.41$ & $<0.001 \backslash$ \\
\hline & NSTEMI & 51 & $6(11.8 \%)$ & $45(88.2 \%)$ & & $41.15 \pm 35.58$ & \\
\hline & Unstable Angina & 35 & $6(17.1 \%)$ & $29(82.9 \%)$ & & $30.22 \pm 23.55$ & \\
\hline & Stable Angina & 707 & 237(33.5\%) & $470(66.5 \%)$ & & $24.05 \pm 28.07$ & \\
\hline & Others & 138 & $56(40.6 \%)$ & $82(59.4 \%)$ & & $20.94 \pm 25.05$ & \\
\hline \multirow[t]{2}{*}{$W C(\mathrm{~cm})$} & $<102$ or $<88$ & 367 & $89(24.3 \%)$ & 278(75.7\%) & $<0.001$ & $27.73 \pm 26.98$ & 0.050 \\
\hline & $>=102$ or $>=88$ & 641 & $225(35.1 \%)$ & $416(64.9 \%)$ & & $24.11 \pm 28.90$ & \\
\hline \multirow[t]{2}{*}{ W/HR } & $<=0.9$ or $<=0.85$ & 28 & 10(35.7\%) & 18(64.3\%) & 0.597 & $15.92 \pm 15.11$ & 0.071 \\
\hline & $>0.9$ or $>0.85$ & 980 & $304(31.0 \%)$ & $676(69.0 \%)$ & & $25.70 \pm 28.50$ & \\
\hline \multirow[t]{2}{*}{$\mathrm{W} / \mathrm{HtR}$} & $<0.5$ & 60 & $14(23.3 \%)$ & $46(76.7 \%)$ & 0.178 & $25.62 \pm 24.34$ & 0.956 \\
\hline & $>=0.5$ & 948 & $300(31.6 \%)$ & $648(68.4 \%)$ & & $25.42 \pm 28.50$ & \\
\hline \multirow[t]{2}{*}{ BMI $\left(\mathrm{kg} / \mathrm{m}^{2}\right)$} & $<25$ & 226 & $53(23.5 \%)$ & $173(76.5 \%)$ & $<0.001$ & $27.15 \pm 27.00$ & 0.015 \\
\hline & $25-29$ & 465 & 137(29.5\%) & $328(70.5 \%)$ & & $27.18 \pm 28.65$ & \\
\hline
\end{tabular}


Table 3 Anthropometric variables of the study population as divided by Gensini score groups (Continued)

\begin{tabular}{|c|c|c|c|c|c|c|c|}
\hline \multirow[t]{2}{*}{ Variables } & & \multirow[t]{2}{*}{ Total } & \multicolumn{3}{|c|}{ Gensini score; n(\%) } & \multirow[t]{2}{*}{ Mean \pm SD } & \multirow[t]{2}{*}{$\boldsymbol{P}$-Value } \\
\hline & & & $<7$ & $>=7$ & $\boldsymbol{P}$-value & & \\
\hline & $>=30$ & 317 & $124(39.1 \%)$ & 193(60.9\%) & & $21.62 \pm 28.26$ & \\
\hline \multirow[t]{2}{*}{ HLP ((frequency, percentile) } & Yes & 234 & $57(24.4 \%)$ & 177(75.6\%) & 0.010 & $31.11 \pm 31.65$ & $<0.001$ \\
\hline & No & 774 & $257(33.2 \%)$ & $517(66.8 \%)$ & & $23.71 \pm 26.93$ & \\
\hline
\end{tabular}

HTN Hypertension, DM Diabetes mellitus, CABG Coronary artery bypass graft, $P C I$ Percutaneous coronary intervention, $A C S$ Acute coronary syndrome, $H F$ Heart failure, FH Family history, HRT Hormone replacement therapy, MI Myocardial infarction, STEMI ST-elevation myocardial infarction, NSTEMI Non ST-elevation myocardial infarction, WC Waist circumference, W/HR Waist to hip ratio, W/HtR Waist to height ratio, BMI Body mass index, $H L P$ Hyperlipidemia, CAD Coronary artery disease

$P=0.002$, and $29.3 \%$ vs. $10.7 \%, P=0.032$, respectively) (Table 6).

\section{Discussion}

In this study, it was observed that the patients with higher SYNTAX and Gensini scores were older with a higher prevalence of DM, HTN, and HLP, compared to those with lower scores. The subjects with higher SYNT AX and Gensini scores also had a lower WC and BMI and showed a higher probability of undergoing coronary angiography due to ACS or HF than the patients with lower scores. A significant direct correlation was also observed between the Gensini and SYNTAX scores with age and an inverse correlation with LVEF, BMI, WC, and WHtR. After the modulation of the confounding effects of other variables, male gender, age, DM, HLP, and NSTEMI appeared to predict the extent of coronary atherosclerosis. However, BMI and other indices of abdominal obesity did not show to be the predictors of severe CAD.

Based on the literature, it was shown that the anthropometric indices of obesity and overweightness have been associated with higher cardiometabolic risk and were reported to have good predictive values for

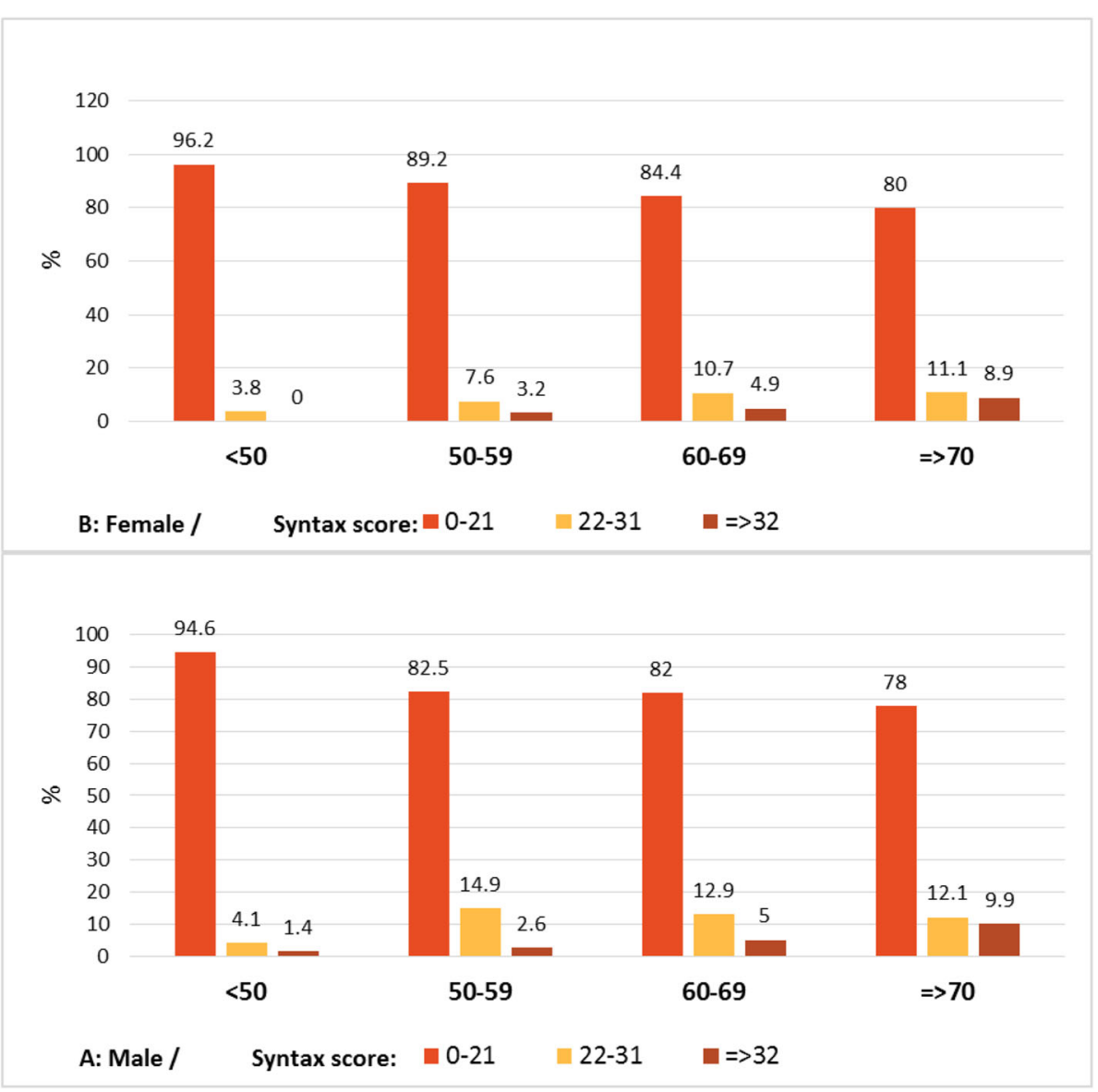

Fig. 1 The severity of CAD expressed by SYNTAX score in different age groupscategorized by sex. 


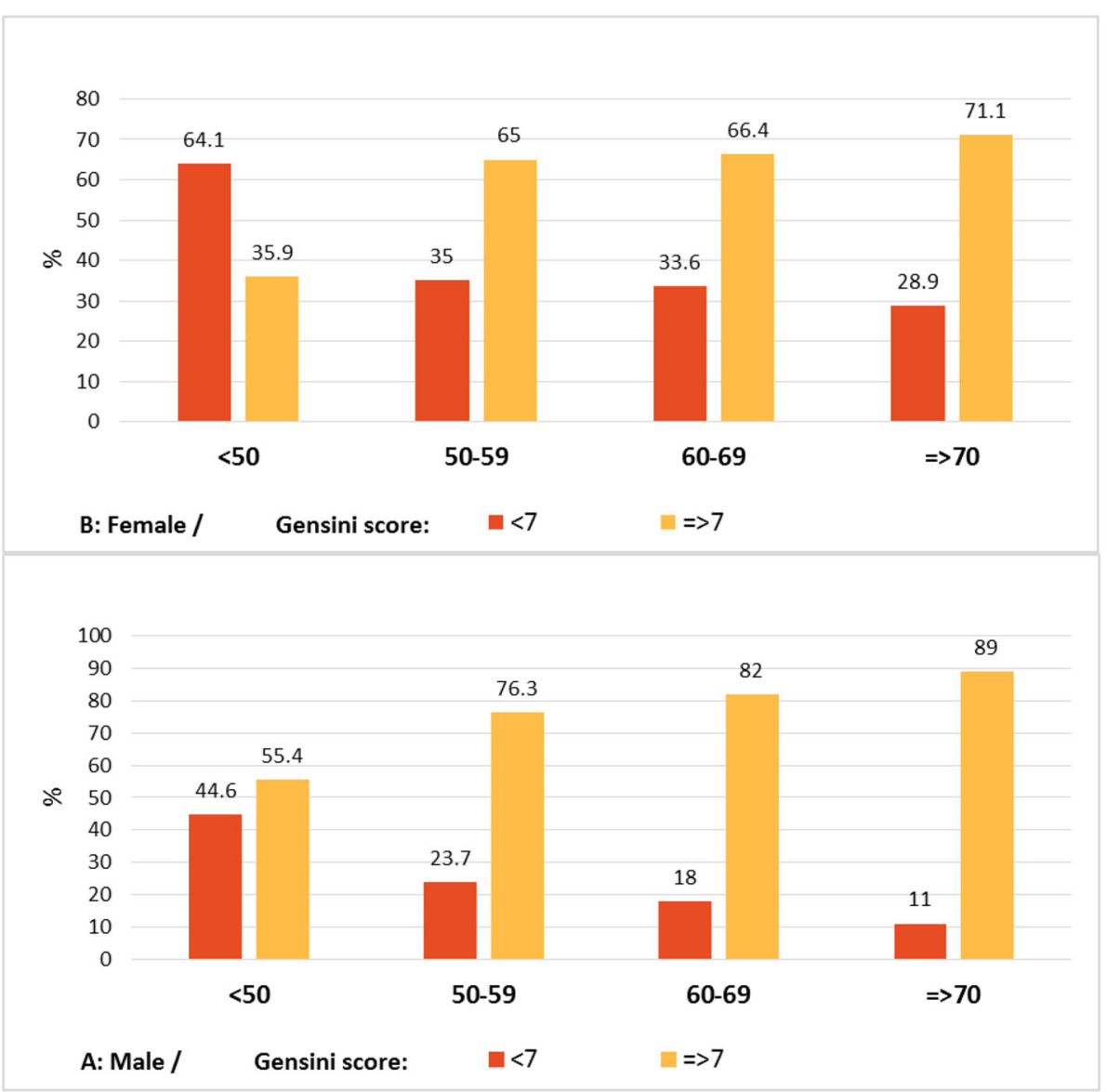

Fig. 2 The severity of CAD expressed by gensini score in different age groups categorized by sex

assessing the probability of CAD [2]. Although BMI as an index of obesity is linked to the increased risk of cardiovascular disease, it seems that the pattern of body fat distribution is a more important determinant of risk than BMI [19].

The WHtR is an effective abdominal obesity index that has been proved to have a robust association with cardiovascular risk factors in Asian population and predicts the risk of diabetes and coronary heart diseases in the general population [17]. Due to the adverse effects of obesity on cardiovascular risk factors, as well as the structure and function of the heart, the prevalence of all cardiovascular diseases increased in the setting of obesity. However, many studies using the various measurements of obesity demonstrated an obesity paradox in patients with CAD with good prognosis among overweight and obese patients with CAD [5].

Rubinshtein et al. evaluated the correlation between BMI, extent of CAD, and prevalence of high-risk coronary anatomy in 928 patients who underwent coronary angiography. It was observed that obese subjects were younger with a lower prevalence of high-risk coronary anatomy. Obese patients were probably referred for angiography earlier than nonobese cases, and this may explain the obesity paradox in these subjects [20]. In another study, the BMI was compared among 842 patients with and without angiographic CAD who underwent coronary angiography. The subjects with coronary stenosis $>50 \%$ were less likely to be obese and more likely to be at ideal body weight, compared to the patients with a lower degree of coronary stenosis [4].

Again, another cohort study evaluated 1299 patients who had undergone coronary angiography to determine if there was a significant correlation between BMI with the extent of coronary atherosclerosis, coronary events, and mortality. The Gensini score was used to determine the burden of coronary atherosclerosis. Overweight and obese subjects had a higher prevalence of HLP, HTN, and DM, compared to normal-weight patients; however, BMI was not significantly associated with a higher extent of coronary atherosclerosis. Mortality due to cardiac events was not different between groups. However, obese and overweight patients had a higher incidence of coronary events, compared to normal-weight patients [21].. This finding is similar to the results in the present study that the various indices of obesity were not the 
Table 4 Multivariate predictors of atherosclerosis severity (SYNT AX score)

\begin{tabular}{|c|c|c|c|}
\hline Variables & & $\beta$ & $\boldsymbol{P}$-value \\
\hline \multirow[t]{2}{*}{ Gender } & Male & - & - \\
\hline & Female & -0.09 & 0.020 \\
\hline \multirow[t]{2}{*}{ HTN } & Yes & 0.04 & 0.146 \\
\hline & No & - & - \\
\hline \multirow[t]{2}{*}{ DM } & Yes & 0.09 & 0.002 \\
\hline & No & - & - \\
\hline \multirow[t]{2}{*}{ ACS or HF } & Yes & -0.01 & 0.722 \\
\hline & No & - & - \\
\hline \multirow[t]{2}{*}{ HRT } & Yes & -0.02 & 0.484 \\
\hline & No & - & - \\
\hline \multirow[t]{2}{*}{ Ml } & Yes & 0.07 & 0.045 \\
\hline & No & - & - \\
\hline \multirow[t]{5}{*}{ Type of presentation } & STEMI & 0.06 & 0.146 \\
\hline & NSTEMI & 0.13 & $<0.001$ \\
\hline & Unstable Angina & 0.07 & 0.033 \\
\hline & Stable Angina & 0.03 & 0.379 \\
\hline & Others & - & - \\
\hline \multirow[t]{2}{*}{ HLP } & Yes & 0.14 & $<0.001$ \\
\hline & No & - & - \\
\hline Age & & 0.19 & $<0.001$ \\
\hline LVEF & & -0.15 & $<0.001$ \\
\hline WC & & 0.003 & 0.970 \\
\hline $\mathrm{WHtR}$ & & 0.01 & 0.897 \\
\hline BMI & & -0.06 & 0.290 \\
\hline
\end{tabular}

HTN Hypertension, DM Diabetes mellitus, ACS Acute coronary syndrome, HF Heart failure, HRT Hormone replacement therapy, MI Myocardial infarction, STEMI ST-elevation myocardial infarction, NSTEMI Non ST-elevation myocardial infarction, WC Waist circumference, WHtR Waist to height ratio, BMI: Body mass index, HLP Hyperlipidemia, LVEF Left ventricular ejection fraction

predictors of the extent of coronary atherosclerosis in spite of being associated with cardiovascular risk factors, such as aging, HLP, and DM. Higher incidence of coronary events in obese subjects is probably due to associated cardiovascular risk factors, impaired endothelial function, and inflammation [21]. Paradoxical association of obesity with a lower burden of coronary atherosclerosis may be partly due to the limitations of noninvasive studies to accurately diagnose the severity of CAD in obese patients resulting in the referral of the subjects without disease for angiography at an earlier time [22].

On the other hand, a low BMI may indicate a low level of serum cholesterol, triglyceride, total fat-free mass, and other anthropometric indices, such as a small thigh circumference, which has been related to the total mortality [23]. Severe CAD is associated with a lower LVEF, compared to single-vessel or two-vessel disease. Reduced LVEF is considered an important parameter of
Table 5 Multivariate predictors of atherosclerosis severity (Gensini score)

\begin{tabular}{|c|c|c|c|}
\hline Variables & & $\beta$ & $P$-value \\
\hline \multirow[t]{2}{*}{ Gender } & Male & - & - \\
\hline & Female & -0.11 & 0.010 \\
\hline \multirow[t]{2}{*}{ HTN } & Yes & 0.03 & 0.343 \\
\hline & No & - & - \\
\hline \multirow[t]{2}{*}{ DM } & Yes & 0.12 & $<0.001$ \\
\hline & No & - & - \\
\hline \multirow[t]{2}{*}{ ACS or HF } & Yes & 0.01 & 0.691 \\
\hline & No & - & - \\
\hline \multirow[t]{2}{*}{ HRT } & Yes & -0.03 & 0.300 \\
\hline & No & - & - \\
\hline \multirow[t]{2}{*}{ Ml } & Yes & 0.10 & 0.003 \\
\hline & No & - & - \\
\hline \multirow[t]{5}{*}{ Type of presentation } & STEMI & 0.008 & 0.841 \\
\hline & NSTEMI & 0.11 & 0.002 \\
\hline & Unstable Angina & 0.009 & 0.760 \\
\hline & Stable Angina & 0.02 & 0.618 \\
\hline & Others & - & - \\
\hline \multirow[t]{2}{*}{ HLP } & Yes & 0.14 & $<0.001$ \\
\hline & No & - & - \\
\hline Age & & 0.18 & $<0.001$ \\
\hline EF & & -0.16 & $<0.001$ \\
\hline WC & & 0.02 & 0.809 \\
\hline $\mathrm{WHtR}$ & & 0.03 & 0.779 \\
\hline BMI & & -0.07 & 0.212 \\
\hline
\end{tabular}

HTN Hypertension, DM Diabetes mellitus, ACS Acute coronary syndrome, HF Heart failure, HRT Hormone replacement therapy, MI Myocardial infarction, STEMI ST-elevation myocardial infarction, NSTEMI Non ST-elevation myocardial infarction, WC Waist circumference, W/HtR Waist to height ratio, BMI Body mass index, HLP Hyperlipidemia, LVEF Left ventricular ejection fraction

identifying high-risk patients, who are most likely to benefit from a more aggressive treatment [24]. This finding is consistent with the results of the present study that the patients with a more extensive CAD had a lower LVEF, compared to the subjects with a lower level of extensive disease.

In the present study, the subjects with NSTEMI had higher SYNTAX and Gensini scores in comparison to the patients with STEMI. This result is consistent with the findings of previous studies indicating that the majority of culprit lesions in STEMI were associated with a less complex structure, compared to those causing NSTEMI [25].

\section{Limitation}

In our study, echocardiographic assessment of LVEF was not performed independently by two researchers. However, evaluation of echocardiographic data was not the 
Table 6 Comparison of anthropometric indices among patients with diabetes, blood pressure and coexistence of DM and HTN

\begin{tabular}{|c|c|c|c|c|c|c|c|c|c|c|c|}
\hline \multirow[t]{2}{*}{ Variables } & & \multirow[t]{2}{*}{ Total } & \multicolumn{2}{|l|}{ DM; n (\%) } & \multirow[t]{2}{*}{$P$} & \multicolumn{2}{|l|}{ HTN; n (\%) } & \multirow[t]{2}{*}{$P$} & \multicolumn{2}{|c|}{ DM and HTN; n (\%) } & \multirow[t]{2}{*}{$P$} \\
\hline & & & Yes $=391$ & $\mathrm{No}=617$ & & Yes $=635$ & $\mathrm{No}=373$ & & Yes $=290$ & $\mathrm{No}=718$ & \\
\hline \multirow[t]{2}{*}{ WC } & $<102$ or $<88$ & 367 & $121(33)$ & $246(67)$ & 0.004 & 195(53.1) & $172(46.9)$ & $<0.001$ & $84(22.9)$ & 283(77.1) & 0.002 \\
\hline & $>=102$ or $>=88$ & 641 & $270(42.1)$ & $371(57.9)$ & & $440(68.6)$ & 201(31.4) & & 206(32.1) & $435(67.9)$ & \\
\hline \multirow[t]{2}{*}{ W/HR } & $<=0.9$ or $<=0.85$ & 28 & $8(28.6)$ & $20(71.4)$ & 0.260 & $11(39.3)$ & $17(60.7)$ & 0.008 & $3(10.7)$ & $25(89.3)$ & 0.032 \\
\hline & $>0.9$ or $>0.85$ & 980 & $383(39.1)$ & $597(60.9)$ & & $624(63.7)$ & $356(36.3)$ & & $287(29.3)$ & $693(70.7)$ & \\
\hline \multirow[t]{2}{*}{$\mathrm{W} / \mathrm{HtR}$} & $<0.5$ & 60 & $24(40)$ & $36(60)$ & 0.843 & $33(55)$ & $27(45)$ & 0.186 & $17(28.3)$ & $43(71.7)$ & 0.939 \\
\hline & $>=0.5$ & 948 & $367(38.7)$ & $581(61.3)$ & & $602(63.5)$ & $346(36.5)$ & & 273(28.8) & $675(71.2)$ & \\
\hline \multirow[t]{3}{*}{ BMI } & $<25$ & 226 & $76(33.6)$ & $150(66.4)$ & 0.194 & 132(58.4) & $94(41.6)$ & 0.038 & $54(23.9)$ & $172(76.1)$ & 0.183 \\
\hline & $25-29$ & 465 & 188(40.4) & 277(59.6) & & $286(61.5)$ & 179(38.5) & & $141(30.3)$ & $324(69.7)$ & \\
\hline & $>=30$ & 317 & $127(40.1)$ & $190(59.9)$ & & $217(68.5)$ & $100(31.5)$ & & $95(30)$ & $222(70)$ & \\
\hline
\end{tabular}

HTN Hypertension, DM Diabetes mellitus, WC Waist circumference, W/HR Waist to hip ratio, W/HtR Waist to height ratio, BMI Body mass index

main purpose of this study. Also, our study was crosssectional and the duration of overweightness which may influence the results was not included in the study. Thus, this study could not show any causal relationship between obesity and the severity of CAD.

\section{Conclusion}

In conclusion, it seems that the anthropometric indices of obesity are not the predictors of the severity of CAD. However, the anthropometric indices of obesity are associated with the increased risk of cardiovascular risk factors and high-risk profile.

\footnotetext{
Abbreviations

CAD: Coronary artery disease; BMI: Body mass index; WC: Waist circumference; WHR: Waist-to-hip ratio; WHtR: Waist-to-height ratio; DM: Diabetes mellitus; HTN: Hypertension; HR: Heart failure; WHO: World Health Organization; ESC: European society of cardiology; LVEF: Left ventricular ejection fraction; LVESV: Left ventricular end-systolic volume; LVEDV: Left ventricular end-diastolic volume; CABG: Coronary artery bypass graft; PCl: Percutaneous coronary intervention; ACS: Acute coronary syndrome; HRT: Hormone replacement therapy; MI: Myocardial infarction; STEMI: ST-elevation myocardial infarction; Non STEMI: Non ST-elevation myocardial infarction; HLP: Hyperlipidemia
}

\section{Acknowledgments}

The present study was extracted from a postgraduate thesis written by Dr. Ehsan Soroosh. The authors would like to thank all the patients and hospital staff for their care and support.

\section{Authors' contributions}

$M N, A G, E S$ and MM acquired data, performed the statistical analyses, interpreted data, and drafted and revised the manuscript for important intellectual content and approved the final version. HS, and MG interpreted data and revised the manuscript for important intellectual content and approved the final version. All authors have read and approved the manuscript.

\section{Funding}

This study was supported by research deputy of Mazandaran University of Medical Science. The funder had no role in the design of the study, data collection, analysis, interpretation of data, and writing of the manuscript or decision to publish.

\section{Availability of data and materials}

The datasets used and/or analysed during the current study are available from the corresponding author on reasonable request.

\section{Ethics approval and consent to participate}

This study was approved by Mazandaran University of Medical science ethical committee (IR.MAZUMS.REC.1396.10251). Also, written informed consent was obtained from all participants.

\section{Consent for publication}

Not applicable.

\section{Competing interests}

The authors declare that they have no Competing interests.

\section{Author details}

${ }^{1}$ Cardiovascular Research Center, Mazandaran University of Medical Sciences, Sari, Iran. ${ }^{2}$ Health Sciences Research Center, Addiction Institute, Mazandaran University of Medical Sciences, Sari, Iran. ${ }^{3}$ Department of Cardiology,

Golestan University of Medical Sciences, Gorgan, Iran. ${ }^{4}$ Mazandaran Heart Center, Artesh BLVD, Sari, Iran.

Received: 29 January 2020 Accepted: 17 May 2020

Published online: 29 May 2020

\section{References}

1. Williams EP, Mesidor M, Winters K, Dubbert PM, Wyatt SB. Overweight and obesity: prevalence, consequences, and causes of a growing public health problem. Curr Obes Rep. 2015:4(3):363-70.

2. Ashwell M, Gunn P, Gibson S. Waist-to-height ratio is a better screening tool than waist circumference and BMI for adult cardiometabolic risk factors: systematic review and meta-analysis. Obes Rev. 2012;13(3):275-86.

3. Huxley R, Mendis S, Zheleznyakov E, Reddy S, Chan J. Body mass index, waist circumference and waist: hip ratio as predictors of cardiovascular risk—a review of the literature. Eur J Clin Nutr. 2010;64(1):16.

4. Phillips SD, Roberts WC. Comparison of body mass index among patients with versus without angiographic coronary artery disease. Am J Cardiol. 2007;100(1):18-22

5. Lavie CJ, De Schutter A, Parto P, Jahangir E, Kokkinos P, Ortega FB, et al. Obesity and prevalence of cardiovascular diseases and prognosis - the obesity paradox updated. Prog Cardiovasc Dis. 2016;58(5):537-47.

6. Chobanian AV, Bakris GL, Black HR, Cushman WC, Green LA, Izzo JL Jr, et al. Seventh report of the joint National Committee on prevention, detection, evaluation, and treatment of high blood pressure. Hypertension. 2003;42(6):1206-52.

7. Association AD. Diagnosis and classification of diabetes mellitus. Diab Care. 2008:31(Supplement 1):S55-60.

8. Parmar MS. Family history of coronary artery disease-need to focus on proper definition. Eur Heart J. 2003;24(22):2073. 
9. Wood D, De Backer G, Faergeman O, Graham I, Mancia G, Pyorala K. Prevention of coronary heart disease in clinical practice. Summary of recommendations of the second joint task force of European and other societies on coronary prevention. J Hypertens. 1998;16(10):1407-14.

10. Klein S, Allison DB, Heymsfield SB, Kelley DE, Leibel RL, Nonas C, et al. Waist circumference and cardiometabolic risk: a consensus statement from shaping America's health: Association for Weight Management and Obesity Prevention; NAASO, the Obesity Society; the American Society for Nutrition; and the American Diabetes Association. Obesity. 2007;15(5):1061-7.

11. Garnett S, Baur L, Cowell C. Waist-to-height ratio: a simple option for determining excess central adiposity in young people. Int J Obes. 2008; 32(6):1028.

12. Members ATF, Hamm CW, Bassand J-P, Agewall S, Bax J, Boersma E, et al. ESC guidelines for the management of acute coronary syndromes in patients presenting without persistent ST-segment elevation: the task force for the management of acute coronary syndromes (ACS) in patients presenting without persistent ST-segment elevation of the European Society of Cardiology (ESC). Eur Heart J. 2011;32(23):2999-3054.

13. Members ATF, Steg PG, James SK, Atar D, Badano LP, Lundqvist CB, et al. ESC guidelines for the management of acute myocardial infarction in patients presenting with ST-segment elevation: the task force on the management of ST-segment elevation acute myocardial infarction of the European Society of Cardiology (ESC). Eur Heart J. 2012;33(20):2569-619.

14. Ponikowski P, Voors AA, Anker SD, Bueno H, Cleland JG, Coats AJ, et al. 2016 ESC guidelines for the diagnosis and treatment of acute and chronic heart failure: the task force for the diagnosis and treatment of acute and chronic heart failure of the European Society of Cardiology (ESC). Developed with the special contribution of the heart failure association (HFA) of the ESC. Eur J Heart Fail. 2016;18(8):891-975.

15. Harris PJ, Behar VS, Conley MJ, Harrell FE, Lee KL, Peter RH, et al. The prognostic significance of $50 \%$ coronary stenosis in medically treated patients with coronary artery disease. Circulation. 1980;62(2):240-8.

16. Sabah KMN, Chowdhury AW, Khan HLR, Hasan AH, Haque S, Ali S, et al. Body mass index and waist/height ratio for prediction of severity of coronary artery disease. BMC Res Notes. 2014;7(1):246.

17. Avci A, Fidan S, Tabakçı MM, Toprak C, Alizade E, Acar E, et al. Association between the Gensini score and carotid artery stenosis. Korean Circ J. 2016; 46(5):639-45.

18. Gannot S, Fefer P, Kopel E, Kochkina K, Beigel R, Raanani E, et al. Higher Syntax score is not predictive of late mortality in "real-world" patients with multivessel coronary artery disease undergoing coronary artery bypass grafting. Israel Med Assoc J. 2014;16(12):764-7.

19. Mirmiran P, Esmaillzadeh A, Azizi F. Detection of cardiovascular risk factors by anthropometric measures in Tehranian adults: receiver operating characteristic (ROC) curve analysis. Eur J Clin Nutr. 2004;58(8):1110.

20. Rubinshtein R, Halon DA, Jaffe R, Shahla J, Lewis BS. Relation between obesity and severity of coronary artery disease in patients undergoing coronary angiography. Am J Cardiol. 2006:97(9):1277-80.

21. Rossi R, laccarino D, Nuzzo A, Chiurlia E, Bacco L, Venturelli A, et al. Influence of body mass index on extent of coronary atherosclerosis and cardiac events in a cohort of patients at risk of coronary artery disease. Nutr Metab Cardiovasc Dis. 2011;21(2):86-93.

22. Stalls CM, Triplette MA, Viera AJ, Pathman DE, Cohen MG, Rossi JS. The association between body mass index and coronary artery disease severity: a comparison of black and white patients. Am Heart J. 2014;167(4):514-20.

23. Chen Y, Copeland WK, Vedanthan R, Grant E, Lee JE, Gu D, et al. Association between body mass index and cardiovascular disease mortality in east Asians and south Asians: pooled analysis of prospective data from the Asia cohort consortium. Bmj. 2013;347:f5446.

24. Squeri A, Gaibazzi N, Reverberi C, Caracciolo MM, Ardissino D, Gherli T. Ejection fraction change and coronary artery disease severity: a vasodilator contrast stress-echocardiography study. J Am Soc Echocardiogr. 2012;25(4):454-9.

25. Zaman T, Agarwal S, Anabtawi AG, Patel NS, Ellis SG, Tuzcu EM, et al. Angiographic lesion severity and subsequent myocardial infarction. Am J Cardiol. 2012:110(2):167-72.

\section{Publisher's Note}

Springer Nature remains neutral with regard to jurisdictional claims in published maps and institutional affiliations.

\section{Ready to submit your research? Choose BMC and benefit from}

- fast, convenient online submission

- thorough peer review by experienced researchers in your field

- rapid publication on acceptance

- support for research data, including large and complex data types

- gold Open Access which fosters wider collaboration and increased citations

- maximum visibility for your research: over $100 \mathrm{M}$ website views per year

At $\mathrm{BMC}$, research is always in progress.

Learn more biomedcentral.com/submissions 